\title{
ERRATUM
}

Open Access

\section{Erratum to: A study of the deterioration of aged parchment marked with laboratory iron gall inks using FTIR-ATR spectroscopy and micro hot table}

Stamatis C. Boyatzis", Georgia Velivasaki and Ekaterini Malea

\section{Erratum to: Herit Sci (2016) 4:13}

DOI 10.1186/s40494-016-0083-4

After publication of the original article [1], the authors found an error with Table 1, the types of vibrations for sulphates were input incorrectly; i.e. $v_{3} \mathrm{SO}_{4}{ }^{2-}$ should be $v_{1} \mathrm{SO}_{4}{ }^{2-}$ and $v_{1} \mathrm{SO}_{4}{ }^{2-}$ should be $v_{3} \mathrm{SO}_{4}{ }^{2-}$. Please see the correct table in this erratum (Table 1 ). 
Table 1 Assignment of main infrared peaks $\left(\mathrm{cm}^{-1}\right)$ of standard materials and compounds

\begin{tabular}{|c|c|c|}
\hline Absorption maximum $\left(\mathrm{cm}^{-1}\right)$ & & Assignment \\
\hline \multicolumn{3}{|l|}{ Collagenous materials } \\
\hline Collagen & Parchment & \\
\hline $3315 \mathrm{~s}, \mathrm{br}$ & 3302 & $\begin{array}{l}\text { Amide A: first component of } v \mathrm{~N}-\mathrm{H} \text { in Fermi resonance with the amide } \\
\text { II overtone (overlapping with } v_{a s} \mathrm{O}-\mathrm{H}(3447 \mathrm{br}) \text { and } v_{s} \mathrm{O}-\mathrm{H}(3240 \mathrm{br}) \text { of } \\
\text { structural } \mathrm{H}_{2} \mathrm{O} \text { ) }\end{array}$ \\
\hline $3072, \mathrm{~m}-\mathrm{w}, \mathrm{br}$ & 3072 & $\begin{array}{l}\text { Amide B: (second component of } \mathrm{vN}-\mathrm{H} \text { in Fermi resonance with the } \\
\text { amide II overtone) }\end{array}$ \\
\hline 2958 & 2926 & $V C-H$ \\
\hline $1640 \mathrm{~s}$ & 1644 & $\begin{array}{l}\text { Amide l: } v C=\mathrm{O} \text { with small contributions from } v C-\mathrm{N} \text { and } \delta \mathrm{N}-\mathrm{H} \text { ) [overlap- } \\
\left.\text { ping with } \delta \mathrm{H}-\mathrm{O}-\mathrm{H} \text { (approx. 1610) of structural } \mathrm{H}_{2} \mathrm{O}\right]^{\mathrm{a}}\end{array}$ \\
\hline $1545 \mathrm{~s}$ & 1538 & Amide II: $\left(v C-N \text { with contributions from } \delta_{i p} \mathrm{~N}-\mathrm{H}\right)^{\mathrm{b}}$ \\
\hline $1454 \mathrm{~m}-\mathrm{w}$ & 1448 & $\delta \mathrm{CH}_{2}$ of Pro- \\
\hline $1405 w$ & 1408 & $\delta_{i p} \mathrm{C}-\mathrm{O}-\mathrm{H}$ (carboxylic side chains) and $\delta \mathrm{NH}_{2}$ \\
\hline $1340 w$ & 1334 & $\mathrm{wCH} / \mathrm{C}-\mathrm{H}$ (methine) \\
\hline $1241 \mathrm{~m}-\mathrm{w}$ & 1230 & Amide III: $\left(v C-N+\delta N-H \text { with contributions from } v C-C \text { and } \delta_{i p} C=O\right)^{b}$ \\
\hline 1082,1032 & 1084,1031 & $\begin{array}{l}\text { Breathing of proline ring [68] with carbohydrate } \mathrm{vC}-\mathrm{O} \text { and } \mathrm{vC}-\mathrm{O}-\mathrm{C} \\
\text { (glycosylation sites) [69]/parchment: additional esters [70] }\end{array}$ \\
\hline \multicolumn{3}{|l|}{ Gallic acid $[40,73,74]$} \\
\hline 3498 & & $\mathrm{vO}_{9} \mathrm{H}, \mathrm{vO}_{11} \mathrm{H}$ \\
\hline 3366 & & $\mathrm{vO}_{10} \mathrm{H}$ \\
\hline 3284 & & $\mathrm{VO}_{14} \mathrm{H}(\mathrm{COOH})$ \\
\hline $3065,2996,2844$ & & $v \mathrm{C}_{2}-\mathrm{H}_{1}, \mathrm{C}_{6}-\mathrm{H}$ \\
\hline $2673,2632,2575,2512$ & & $\mathrm{VO}_{14} \mathrm{H}$ (-COOH dimers) \\
\hline 1703 & & $v C=O$ \\
\hline 1668,1648 & & $v \mathrm{C}=\mathrm{O}(-\mathrm{COOH}$ dimers $)$ \\
\hline $1612,1542,1484,1468,1427,1387,1321$ & & $v C=C$ (aromatic ring vibrations) \\
\hline 1268 & & $v C-O$ \\
\hline 1221 & & $\delta(i-p) C-O-H$ \\
\hline 1184 & & $v C_{1}-C_{7}+v C-H$ \\
\hline 1099 & & $v \mathrm{C}_{1}-\mathrm{C}_{7}+\delta \mathrm{C}-\mathrm{O}-\mathrm{H}$ \\
\hline 1028 & & Aromatic asym-, sym-breathing, $v C_{1}-C_{7}+v C-O H$ (phenol) \\
\hline 904 & & $v \mathrm{C}-\mathrm{O}+\delta(\mathrm{o}-\mathrm{o}-\mathrm{p}) \mathrm{C}-\mathrm{O}-\mathrm{H}$ (dimer band) $+\delta(\mathrm{o}-\mathrm{o}-\mathrm{p})$ of ring \\
\hline 867 & & $\delta(i-p) C-H$ \\
\hline 767 & & $\delta(o-o-p) C-H$ (out-of-phase) \\
\hline 735 & & $\delta(o-o-p) C-H$ (out-of-phase) $+T C-O H$ (torsion) \\
\hline 703 & & Aromatic ring puckering \\
\hline 636 & & Aromatic ring puckering $+\delta(\mathrm{o}-\mathrm{o}-\mathrm{p}) \mathrm{C}-\mathrm{O}-\mathrm{H}$ (phenolic) \\
\hline 559 & & $\delta(\mathrm{o}-\mathrm{o}-\mathrm{p}) \mathrm{C}-\mathrm{O}-\mathrm{H}$ (phenolic) \\
\hline 492 & & $\tau C$-aromatic ring \\
\hline \multicolumn{3}{|l|}{ Gum Arabic } \\
\hline 3352 & & $\mathrm{VOH}$ \\
\hline 2932 & & $V C-H$ \\
\hline 1604 & & $v_{a s} \mathrm{COO}^{-}$ \\
\hline 1418 & & $v_{s} \mathrm{COO}^{-}$ \\
\hline 1146(sh), 1068, 1035(sh) & & $v C-O$ \\
\hline \multicolumn{3}{|l|}{ Iron (II) sulfate heptahydrate } \\
\hline 3336 & & $\mathrm{VOH}$ \\
\hline 1652 & & $\delta \mathrm{OH}$ in water \\
\hline
\end{tabular}


Table 1 continued

Iron (II) sulfate heptahydrate

\begin{tabular}{|c|c|}
\hline $\begin{array}{l}1092 \\
977 \\
619\end{array}$ & $\begin{array}{l}v_{3} \mathrm{SO}_{4}^{2-} \\
v_{7} \mathrm{SO}_{4}^{2-} \\
v_{4}\left(\text { asym-) } \mathrm{SO}_{4}^{2-}\right.\end{array}$ \\
\hline \multicolumn{2}{|l|}{ LIG ink formulation } \\
\hline 3415 & $\mathrm{VOH}$ in water \\
\hline 1645 & $\delta \mathrm{OH}$ in water \\
\hline 1094 & $v_{3} \mathrm{SO}_{4}{ }^{2-}$ \\
\hline 977 & $v_{1} \mathrm{SO}_{4}^{2-}$ \\
\hline 628 & $v_{4}($ asym- $) \mathrm{SO}_{4}^{2-}$ \\
\hline \multicolumn{2}{|c|}{ LIGG ink formulation } \\
\hline 3439 & $\mathrm{vH}-\mathrm{O}$ in polysaccharide (gum Arabic) and water \\
\hline 2931 & $v \mathrm{C}-\mathrm{H}$ \\
\hline 1640 & $\delta \mathrm{OH}$ in water overlapping with $\mathrm{v}_{a s} \mathrm{COO}^{-}$in polysaccharide (gum Arabic) \\
\hline 1424 & $v_{s} \mathrm{COO}^{-}$in gum Arabic \\
\hline 1083 & $v_{3} \mathrm{SO}_{4}^{2-}$ overlapping with $v \mathrm{C}-\mathrm{O}$ in gum Arabic \\
\hline 1146(sh), 1035(sh) & $v C-O$ in gum Arabic \\
\hline 604 & $v_{4}(a s y m-) \mathrm{SO}_{4}{ }^{2-}$ \\
\hline
\end{tabular}

$v$ stretching, $\delta$ bending, $d$ deformation, ip in-plane, sh shoulder

a According to Ref. [24]

b According to Refs. $[24,46]$

The online version of the original article can be found under doi:10.1186/s40494-016-0083-4.

Received: 12 September 2016 Accepted: 12 September 2016 Published online: 04 November 2016

\section{Reference}

1. Boyatzis SC, Velivasaki G, Malea E. A study of the deterioration of aged parchment marked with laboratory iron gall inks using FTIR-ATR spectroscopy and micro hot table. Herit Sci. 2016;4:13. doi:10.1186/ s40494-016-0083-4. 